\title{
DOS ESPECIES NUEVAS DE GALIANTHE (RUBIACEAE) PARA BRASIL
}

\author{
ELSA L. CABRAL
}

\begin{abstract}
Summary: Cabral, E. L. 2004. Two new species of the genus Galianthe (Rubiaceae) from Brazil. Bonplandia 13(1-4): 15-19. ISSN: 0524-0476.

Galianthe longisepala E. L. Cabral and Galianthe matogrossiana E. L. Cabral, new species from Brazil, are described and illustrated.
\end{abstract}

Key words: Taxonomy, Rubiaceae, Galianthe longisepala, Galianthe matogrossiana, Brazil.

Resumen: Cabral, E. L. 2004. Galianthe (Rubiaceae): Dos especies nuevas de Galianthe (Rubiaceae) para Brasil. Bonplandia 13(1-4): 15-19. ISSN: 0524-0476.

Galianthe longisepala E. L. Cabral y Galianthe matogrossiana E. L. Cabral, nuevas especies de Brasil, son descriptas e ilustradas.

Palabras clave: Taxonomía, Rubiaceae, Galianthe longisepala, Galianthe matogrossiana, Brasil.

En la revisión del género americano Galianthe Griseb. (Cabral, 2002 inéd.) se reconocen 49 especies preferentemente sudamericanas. Estos taxones tienen un centro de concentración en Brasil, en el planalto central y en el planalto meridional, con 45 especies de las cuales 20 son exclusivas. El propósito de este trabajo es presentar dos especies nuevas para la ciencia: Galianthe longisepala E. L. Cabral y G. matogrossiana E. L. Cabral.

Galianthe longisepala E. L. Cabral sp. nov. Fig. 1

Suffrutex 15-25 (-30) cm alt., 5-10 caulibus subtetragonis, glabris; foliis 20-45 mm longa $x$ 35 (-8) $\mathrm{mm}$ lata, lanceolatis, elliptico-lanceolatis, glabris; stipulis 1,5-3 $\mathrm{mm}$ longa, glabris, puberulis, 3-5 laciniis, 1,5-6 $\mathrm{mm}$ longa. Thyrsi breves in omnibus ramis. Hypantho 2-2,5 $\mathrm{mm}$ longa, obconico, micropapillato. Calyx 4-partitus, segmentis (2-) 3-4 (-5) mm longa, linear-subulatis vel triangularis, intersegmentis cum appendicibus. Corolla alba, externe micropapillata, intus 2 annulari-pilis moniliformis. Floris brevistyli, corolla 3,8-4 mm longa. Floris longistyli, corolla 4-4,5 mm longa. Disco integer papilloso. Capsula 3,5-5 mm longa, glabra, sepalis persistentibus, reflexus. Semina 2,5-3 $\mathrm{mm}$ longa, ventre sulcato circum strophiolo.

Holotypus: BRASIL. Goiás: Serra dos Cristais, $2 \mathrm{~km} \mathrm{~N}$ of Cristalina, $1250 \mathrm{~m} \mathrm{s.m.,} \mathrm{2-III-1966,}$ H. S. Irwin, J. W. Grear, Jr. R. Souza, R. Reis dos Santos 13307 (UB), isotypi (F!, MO!, NY!, RB!, US!).

Instituto de Botánica del Nordeste, UNNE-CONICET, C.C. 209, 3400 Corrientes, Argentina. Fac. Ci. Exact. y Nat. y Agrim. UNNE, E-mail: ferber@arnet.com.ar 


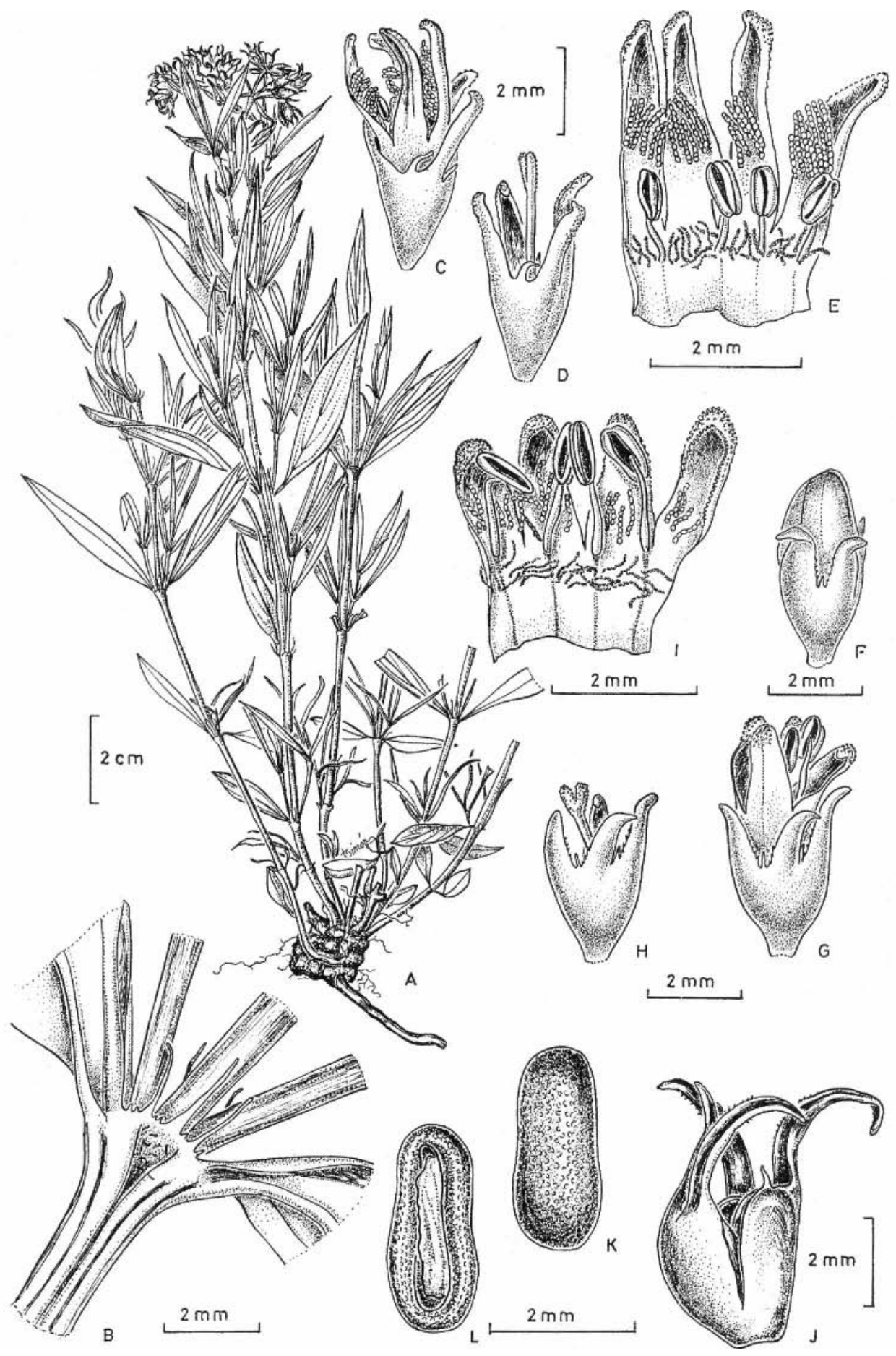

Fig. 1. Galianthe longisepala. A. planta. B: vaina estipular con lacinias. C-E: flor longistila. C: flor. D: hipanto, cáliz, estilo y estigma. E: interior de la corola desplegada. F-H: flor brevistila. F: alabastro. G: flor. H: hipanto, cáliz, estilo y estigma. I: interior de la corola desplegada. J: fruto. K-L: semilla. K: cara dorsal. L: cara ventral. (A-D, J-L, Irwin 13307. F-I, Irwin 25504). Dibujó Laura Simón. 
Sufrútice con xilopodio, de 15-25 (-30) cm alt., ramas simples, 5-10-caule, tallos tetrágonos, glabros, rojizos, 1-2 $\mathrm{mm}$ lat., ramas secundarias poco desarrolladas o ausentes. Hojas 20-45 mm long. x 3-5 (-8) mm lat, desde lanceoladas a elíptico-lanceoladas, glabras en ambas caras, discoloras, con 3-4 pares de nervios secundarios alternos o subopuestos, en material herborizado el color de los nervios en el envés castaño-negruzco, contrasta con el resto de la superficie foliar, nervios terciarios inconspicuos; vaina estipular de $1,5-3 \mathrm{~mm}$ long., pubérula, con 3-5 lacinias, de 1,5-6 mm long., filiformes. Inflorescencias densas en todas las ramas. Hipanto 2-2,5 $\mathrm{mm}$ long., obcónico, micropapiloso, cáliz con 4-segmentos de (2-)3-4 (-5) mm long., linear-subulados, papilosos en el margen, reflexos en el fruto, con dientes o apéndices intercalares, filiformes. Corola blanca, externamente micropapilosa, en la superficie interna con pelos moniliformes, gruesos y largos en los lóbulos y pelos finos y cortos en la mitad superior del tubo. Flor brevistila: corola 3,8-4 mm long., con los lóbulos iguales o más largos que el tubo; anteras $1 \mathrm{~mm}$ long., filamentos 1-1,3 $\mathrm{mm}$ long, papilosos; estilo 2-2,5 mm long., bífido. Flor longistila: corola 4-4,5 mm long., lóbulos más largos que el tubo; anteras 0,7-1 mm long., filamentos 0,4-0,5 mm long. Disco entero, papiloso. Cápsula 3,5-5 mm long., subglobosa, glabra, con sépalos notables, reflexos. Semillas 2,5-3 mm long., subcilíndricas, foveoladas, cara ventral con surco profundo alrededor del estrofíolo persistente.

Distribución geográfica y hábitat. Galianthe longisepala vive en Brasil, en cerrados, en suelos lateríticos y con afloramientos rocosos entre 1050-1250 m s.m. Aparentemente está restringida al planalto central en los estados de Goiás y Minas Gerais. Florece y fructifica de enero a marzo.

Paratypus: BRASIL. Minas Gerais: Morro das Pedras, ca. $25 \mathrm{~km} \mathrm{NE}$ of Patrocinio, elev. 1050 m s.m., 28-I-1970, H.S Irwin \& al. 25504 (NY, RB, UB, US); Municipio de Patrocinio, Fazenda Grao de Ouro, $18^{\circ} 48^{\prime} 31^{\prime \prime S} 46^{\circ} 58^{\prime} 00^{\prime \prime} \mathrm{W}$, 28-II-1989, R. Mendonça \& al. 1191 (UB).
Observaciones. Galianthe longisepala se reconoce fácilmente por las inflorescencias densas y por los frutos con sépalos largos y reflexos; en material herborizado se diferencia por el color de los nervios en el envés, castaño-negruzco, marcadamente contrastante con el resto de la superficie foliar. Es afín a Galianthe lanceifolia Cabral (Cabral, 1993), de la que se diferencia por sus tallos principales con numerosas ramas secundarias, pubescentes, por sus hojas más anchas, 8-18 mm lat, de indumento variado en el haz y en el envés, nervios no contrastantes y los sépalos triangulares, cortos y erectos, persistentes en el fruto.

\section{Galianthe matogrossiana E. L. Cabral sp. nov.}

Fig. 2

Suffrutex 0,8-1 $m$ alt., 1-2-caulibus, subtetragonis, glabris, ramis brevibus absentibus. Foliis 55-90 mm longax 7-20 mm lata, ellipticis glabris. Vagina stipulari supra paribus foliorum suum prolongata. Thyrsi terminali. Hypantho, turbinato, puberulo. Calyx 4-partitus, segmentis, triangularis, intersegmentis cum appendicibus. Corolla 5-6 mm long., alba, externe micropapillata, lobi dorso apicale dense papilloso, intus corolla, pilis moniliformis. Disco integer piloso. Capsula 4-4,5 $\mathrm{mm}$ long. Semina complanata 3-3,5 $\mathrm{mm}$ long.

Holotypus: BRASIL. Mato Grosso do Sul: Municipio Sidrolandia, Agua Rica, 12-IV-1972, G. Hatschbach 29439 (MBM), isotypi: US 2745683!, US 2835289 !.

Sufrútice con xilopodio, erecto de 0,8-1 m alt.; 1-2-caules, tallo subtetrágono, fistuloso, glabro, sin braquiblastos, entrenudos $5-13 \mathrm{~cm}$ long. Hojas 55-90 mm long. x 1-26 mm lat., elíptico-lanceoladas, atenuada hacia el ápice y hacia la base, glabras o con pelos cortos sobre la mitad inferior de los nervios, con 4-5 pares de nervios secundarios subopuestos, más notables en el envés; vaina estipular prolongada por encima de la separación del par de hojas, de 1,5-2,5 $\mathrm{mm}$ long., pilosa, con una lacinia central más desarrollada de 6-7 $\mathrm{mm}$ long. y 2 (-4) laterales más cortas de 2-5 mm long. Inflorescencia tirsoide terminal. Hipanto 2-2,5 


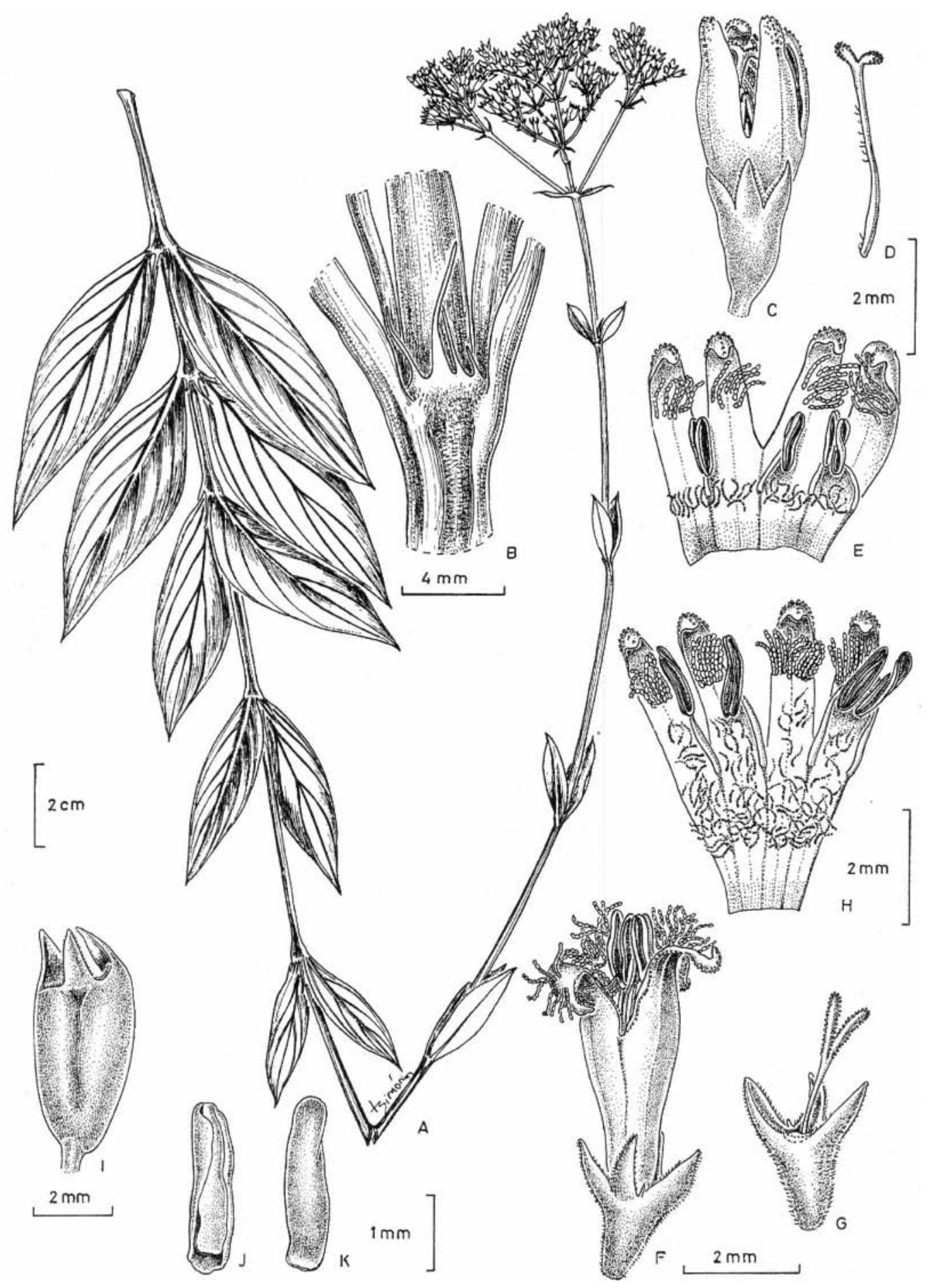

Fig. 2. Galianthe matogrossiana E. L. Cabral. A: planta. B: vaina estipular prolongada por encima de la separación del par de hojas. C-E: flor longistila. C: flor. D: estilo y estigma. E: interior de la corola desplegada. F-H: flor brevistila. F: flor. G: hipanto, cáliz, estilo y estigma. H: interior de la corola desplegada. I: fruto inmaduro. J-K: semilla. J: cara ventral. K: cara dorsal. (A-E, Hatschbach 29439, MBM. F-K, Hatschbach 29439, US). Dibujó Laura Simón. 
mm long., turbinado, pubérulo. Cáliz 4-partido, segmentos del cáliz 1-1,5 mm long., triangulares, dientes intercalares presentes. Corola 5-6 mm long., lóbulos iguales o más largos que el tubo, externamente micropapilosa con papilas cortas y notables en el ápice de los lóbulos. Flor brevistila: interior de la corola con pelos moniliformes, delgados en el tubo y en la base de los lóbulos y pelos gruesos en el tercio superior de los lóbulos, anteras 1,5 mm long., filamentos $2 \mathrm{~mm}$ long.; estilo $2,5 \mathrm{~mm}$, bifurcado en la mitad, ramas estigmáticas lineares. Flor longistila: interior de la corola con pelos moniliformes delgados en la mitad del tubo y pelos moniliformes gruesos en los lóbulos; anteras de $1 \mathrm{~mm}$ long., filamentos de $1,5 \mathrm{~mm}$ long., estilo de 3-3,5 mm long. Disco entero, piloso. Fruto levemente pubescente de 4-4,5 $\mathrm{mm}$ long. Semillas complanadas de 3-3,5 mm long.

Distribución geográfica. Se conocen hasta el presente sólo dos ejemplares de Brasil del estado de Mato Grosso do Sul. Habita en el cerrado.
Paratypus: BRASIL. Mato Grosso do Sul: $22 \mathrm{~km}$ W de Ribas do Rio Pardo, 25-I-1979, A. Krapovickas \& al. 34386 (CTES, SI).

Observaciones. Galianthe matogrossiana pertenece al reducido número de especies que presentan vaina estipular prolongada por encima de la separación del par de hojas. Por esta característica es afín a $G$. guaranitica (Chodat \& Hassl.) E. L. Cabral, pero este taxón presenta hojas pseudoverticiladas, con lámina de base redondeada a levemente cordada y la corola presenta largas papilas en el dorso de los lóbulos.

\section{Bibliografía}

CABRAL, E. L. 1993. Contribución al estudio de los géneros sudamericanos Borreria y Galianthe (Rubiaceae). Bol. Soc. Argent. Bot. 29 (3-4): 225 231.

2002. (inéd.). Revisión del género Galianthe (Rubiaceae). Tesis doctoral, Universidad Nacional del Nordeste, Corrientes.

Original recibido el 16 de junio de 2004; aceptado el 7 de octubre de 2004. 